\title{
Food, Obesity, and Noncommunicable Diseases
}

\author{
Sonali Guleria
}

\begin{abstract}
The scientific and economic development has led on to phenomenal changes in the environment as well as dietary patterns of the human beings. There is a phenomenal increase in the intake of refined carbohydrates, saturated fatty acids (SFAs), trans fat, and substantial decrease in the intake of omega-3 fatty acids. The increased consumption of energy dense food products along with sedentary lifestyle and increased emotional as well as mental stress is an ideal recipe for the development of various noncommunicable diseases (NCD). Increased frequency of combination of environmental, behavioral, and biological risk factors for various NCDs have led on to an epidemic of these diseases in modern times. A healthy food contains adequate amount of carbohydrates with low glycemic index (GI), low sodium content, and sufficient amount of proteins, fibers, micronutrients, and antioxidants. Healthy food and regular exercise are ideal recipes for healthy life and preventing various NCDs. This review discusses the important role of food in various NCDs.

Keywords: Antioxidants, Diet, Noncommunicable diseases, Sodium, Stroke.

Journal of Postgraduate Medicine, Education and Research (2021): 10.5005/jp-journals-10028-1368
\end{abstract}

\section{INTRODUCTION}

Food is essential for our health as it provides us energy and important nutrients required for our well-being and good health. It can also be considered as a medicine as it helps in maintaining good health and also prevents various diseases and in certain instances can also help in treating various diseases. ${ }^{1,2}$ The nutrients present in the food help the cells of our body in preforming various functions. Apart from providing energy and nutrients to the cells of our body, research has also shown that dietary patterns and habits influence the risk of various diseases, especially the noncommunicable diseases (NCDs). Studies have demonstrated that while certain foods and dietary habits may lead to the development of various chronic diseases; others also offer medicinal values that may help in preventing various diseases. ${ }^{1,2}$ In this review, we will discuss the role of diet in causation as well as prevention of various NCDs.

\section{Why Diet is so Important in Causation of NCD in the Current ERa?}

The scientific and economic development has led to phenomenal changes in the environment as well as dietary patterns of the human beings. The human being in the modern times is living in an environment that is remarkably different from our ancestors that lived on the earth in the Paleolithic period approximately 40,000 years ago..$^{1-4}$ The environment and the dietary patterns of the human beings have been almost the same for centuries except for the last 100 years when the dietary patterns and the lifestyle of human beings have undergone a marked transformation. There is a phenomenal increase in the intake of refined carbohydrates, saturated fatty acids (SFAs), trans fat, and substantial decrease in the intake of omega-3 fatty acids. Moreover, all these years, human beings have transformed themselves from eating meat derived from hunting free-roaming animals to eating the meat from grain-fed cattle tamed at farm houses. ${ }^{1-3}$ The studies have shown that over years there has been a marked increase in the amount of calories consumed per person along with the increase in consumption of animal products including meat and dairy products. ${ }^{1-4}$ This increasing pattern of both calories as well as animal products is all
Department of Botany, DAV College, Chandigarh, India

Corresponding Author: Sonali Guleria, Department of Botany, DAV College, Chandigarh, India, Phone: +91 9872968970, e-mail: drsonaliguleria@gmail.com

How to cite this article: Guleria S. Food, Obesity, and Noncommunicable Diseases. J Postgrad Med Edu Res 2021;55(1):8-11.

Source of support: Nil

Conflict of interest: None

across the globe and includes both developed as well as developing countries. The increased consumption of energy dense food products along with sedentary lifestyle and increased emotional as well as mental stress is an ideal recipe for the development of various NCDs (Fig. 1). The energy dense food products apart from providing increased calories also increase the amount of time spent by the human body in the postprandial state. ${ }^{3}$ The modern food products consist of a combination of carbohydrates and fats that provide double peak of increase in blood sugar with the first peak provided by the carbohydrates and the second peak provided

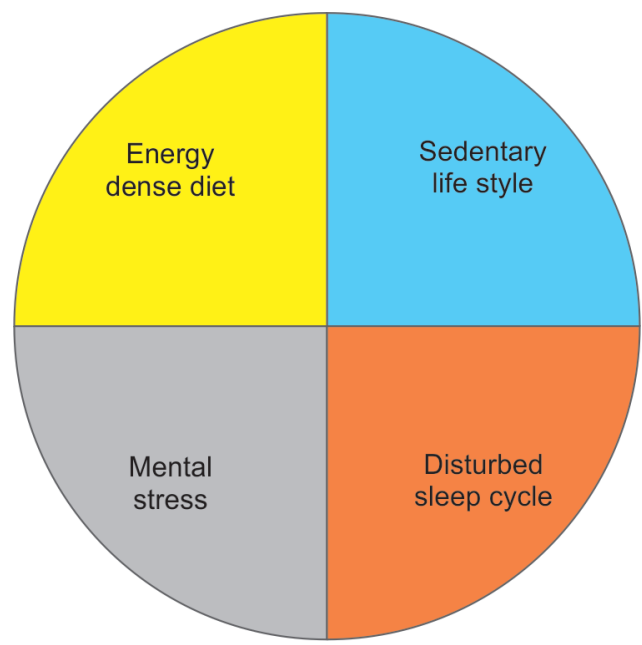

Fig. 1: Factors contributing to noncommunicable diseases 
by the fats. ${ }^{3,5}$ This multipeak pattern increases the time spent by human beings in the postprandial state. The postprandial state produces hyperinsulinemic state and increases the metabolism of glucose as well as fatty acids. The increased metabolism leads to an increased oxidative stress that produces various reactive oxygen species which in turn leads to a proinflammatory state as well as activation of proto-oncogenes that contribute to the development of various NCDs. ${ }^{1-6}$

\section{DieT AND NCDs}

Over the last few decades, the NCDs have emerged as a leading cause of human mortality and morbidity. The important NCDs include diabetes, cardiovascular disease including myocardial infarction and congestive heart failure, chronic respiratory diseases, various cancers, and mental health conditions. It has also been found that obesity and unhealthy lifestyles including eating habits are frequently associated with the presence of one of more of these NCDs. The food we eat in the current era are far less nutritious than we used to eat earlier because of intensive farming and food processing methods, frequent use of pesticides, and use of additives and preservatives.

Increased frequency of combination of environmental, behavioral, and biological risk factors for various NCDs has led on an epidemic of these diseases in modern times. Increased urbanization and globalization combined with low health education and increased mental stress are important environmental factors that have contributed to increased frequency of NCDs. ${ }^{6}$ Increased consumption of alcohol and tobacco, use of unhealthy diet, physical inactivity, and the disruption of circadian rhythm are important behavioral risk factors for the development of NCDs. ${ }^{7,8}$ Apart from the genetic composition, abnormal blood glucose, lipid profile, increased inflammatory state such as increased $C$ reactive protein, increased blood insulin levels, abnormal waist-to-hip ratio, and high blood pressure are important biological risk factors for the development of various NCDs. ${ }^{6-9}$

Studies have shed important light on the role of various dietary patterns and food products in causation as well as prevention of various NCDs. It has been demonstrated that eating predominantly plant-based diets reduces the risk of developing various NCDs such as obesity, diabetes, cardiovascular diseases, and even some forms of cancer. The plant-based diets are the diet that are high in vegetables and fruits, wholegrains, pulses, nuts and seeds, and have only modest amounts of meat and dairy products. ${ }^{8-10}$ This dietary pattern has been shown to maintain a healthy weight and also to reduce blood pressure. Plant-based diets are also rich in dietary fibers that have been shown to protect against colorectal cancer. ${ }^{10}$ On the other hand, eating red and processed meat has shown to increase the risk of developing colorectal cancer. Increased consumption of sodium either in the form of increased salt intake or bakery products has been shown to be a major risk factor for elevated blood pressure and cardiovascular diseases, and also probably contributes in causation of stomach cancer. ${ }^{1-6}$ Diet that is high in meat and dairy products has also been shown to increase the blood pressure. The modern Western food that is high in energy-dense, highly processed foods, and refined starches and/or sugary beverages leads to the development of overweight and obesity. Studies have demonstrated that Western type of diet has adverse effects on the health, whereas Mediterranean diet, Indo-Mediterranean diet, Japanese diet, and vegetarian diet of Europeans may be protective. ${ }^{11,12}$
Diet having increased amount of whole grains has been shown to be related to lower risk of various NCDs, whereas increased consumption of processed meat and unprocessed red meat consumption is associated with increased risk of various NCDs. Studies have also shown that consumption of fruits and vegetables is associated with lower risk of cancer, coronary artery disease, and stroke whereas sugar-sweetened drinks are associated with increased risk of type II diabetes, coronary artery disease, and stroke. ${ }^{13-15}$ Coffee consumption has been found to be protective and has been shown to be associated with lower risk of type II diabetes, nonalcoholic fatty liver disease (NAFLD), and cardiovascular diseases. ${ }^{16}$

\section{Role of Carbohydrates in Causation OF NCDs}

In the field of nutrition, carbohydrate is probably the only macronutrient with no established minimum requirement. However, they have significantly contributed in the increased prevalence of NCDs. The modern starchy foods such as bread, potatoes, and rice raise blood glucose and insulin substantially more than some high sugar foods like whole fruits as they release glucose quickly into the blood stream and therefore are associated with high glycemic index (GI). ${ }^{17}$ On the other hand, some traditionally consumed starches such as legumes and whole kernel grains release the glucose more slowly into the blood stream (low GI). ${ }^{17}$ Various studies have shown that the total amount of carbohydrate consumed as a percentage of dietary energy is less important than the type of carbohydrate consumed for the risk of development of various chronic NCDs. ${ }^{18}$ Foods with high Gl such as refined grains, potatoes, and sugar-sweetened beverages are associated with increased risk of NCDs as they lead to peaks in blood sugar levels and its consequent effects as explained earlier. ${ }^{19}$

The grains are staple foods and major source of dietary carbohydrate for majority of the global population. These are the seeds of cereals and similar plant families and contain three parts: endosperm, bran, and germ. The endosperm is rich in starch, whereas the bran and germ are rich in fiber and proteins/fats, respectively. The minimally processed grains has all three parts, whereas the refined grains available commercially have been processed to remove protein- and fat-rich germ and fiber-rich bran, leaving only the starchy endosperm. ${ }^{20,21}$ It has been shown that there are significant inverse associations between whole grain intake and incidence of type II diabetes, coronary artery disease, ischemic stroke, and several cancers. ${ }^{20,21}$

In certain parts of the world, potatoes are also another major source of dietary carbohydrate. Although they have certain important nutrients such as vitamin C, potassium, and fiber but they predominantly contain starch and that too with high Gl. Studies have shown that increased intake of potatoes is associated with greater weight gain and higher risk of type II diabetes and increased blood pressure. ${ }^{22,23}$ Moreover, fried potatoes leads to further concentration of energy leading to prolonged increase in postprandial hyperglycemia that contributes to obesity and various NCDs. On the other hand, legumes contain low GI carbohydrate and relatively high amounts of protein, fiber, and other nutrients. Population-based study have shown that consumption of diet with increased ratio of beans to white rice was associated with lower cardiometabolic risk factors, including blood lipids and blood pressure. $^{24-26}$ 
Whole fruits are high in fiber, vitamins, minerals, and phytochemicals and typically have moderate to low Gl. Studies have demonstrated that regular consumption of fruits is associated with lower risk of type II diabetes, cardiovascular diseases, cancer, and all-cause mortality. However, this protective effect is limited to consumption of whole fruits and not fruit juices, especially preserved juices, as they are associated with increased $\mathrm{Gl}$ and absence of dietary fibers. ${ }^{27-31}$ Diet also contains important micronutrients that are important in prevention of various NCDs.

\section{Micronutrients AND NCDs}

Inadequate vitamin D and calcium intake are generally seen in aging population and have been shown to be associated with musculoskeletal disorders, such as increased bone fracture risks. Inadequate B vitamins intake and status, in particular folic acid, vitamins $B 6$ and $B 12$, have been associated with age-related cognitive decline as well as memory loss and its supplementation has been shown to improve the cognitive performance. B-vitamins have also been shown to reduce the homocysteine levels and reduced risk for stroke as well as heart attacks. Vitamins $C$ and $D$ have also been shown to reduce the risk of hypertension, and vitamin $\mathrm{D}$ as well as chromium may reduce the risk of diabetes. ${ }^{1-3}$

\section{Diet Intervention Studies and Risk OF NCDs}

Limited evidence suggests that diet intervention can reduce the risk of NCDs. Study comparing paleolithic style diet with standard diet has shown that the paleolithic diet is associated with reduced cardiac mortality as well as decreased frequency of sudden cardiac death as well as overall cardiovascular mortality. Similarly, Indo-Mediterranean diet has been shown to reduce the risk of myocardial infarction, stroke, as well as cardiovascular events. ${ }^{6}$ The Mediterranean diet is the diet followed by people residing in the countries bordering the Mediterranean Sea. This diet is typically high in vegetables, fruits, whole grains, beans, nut and seeds, and olive oil. There is limited intake of red meat in the Mediterranean diet.

\section{SUMMARY}

The food is essential for survival and it is important that the body gets variety of nutrients that are needed for good health and well-being. Whenever possible, an attempt should be made to eat fresh and locally grown foods preferably freshly harvested that are rich in nutrients and fibers and are without preservatives and are not processed. Most of the carbohydrate in our diet should come from unrefined wholegrains that are rich in fibers and have low $\mathrm{Gl}$. One should eat plenty of fruit and vegetables as they provide fiber, micronutrients, and antioxidants, which are essential in the prevention of certain types of cancers and in the reduction of risk for obesity, coronary heart diseases, and various other NCDs. Legumes such as dry beans, peas, lentils, and soya have increased soluble fiber, protein, and flavonoids that contribute significantly to good health. One should not use processed soya products like tofu as an alternative for dry soya beans. Meat, fish, chicken, milk, and eggs can be eaten in moderation as excessive consumption of animal-based food has shown to increase the risk of cancer as well as various other NCDs. The salt and other sodium-containing products should be used sparingly. Use foods and drinks that contain unrefined sugar that is found naturally in fruits and vegetables and one should avoid sweetened drinks. In every food product, one should carefully check the label for nutrition facts. The food product should be providing less calories, minimal saturated and trans fat as well as sodium, and should be high in fibers, vitamins, and minerals. Fast food consumption has been shown to be associated with weight gain and obesity, and this could also increase the risk of NCDs. ${ }^{32}$ They are associated with obesity and NCDs due to extremely high energy density of these foods. ${ }^{33}$

\section{ConCLUSION}

Diet plays a very important role in keeping us healthy and a balanced diet is very essential in protecting us from various NCDs. If food is tasty probably it is high in calories and is processed and therefore increases the risk of NCDs. A healthy food contains adequate amount of carbohydrates with low Gl, low sodium content, and sufficient amount of proteins, fiber, micronutrients, and antioxidants. Healthy food and regular exercise are ideal recipes for healthy life and preventing various NCDs. One should adopt an healthy lifestyle immediately as it is better late than never!

\section{References}

1. Greenberg H, Deckelbaum RJ. Diet and non-communicable diseases: an urgent need for new paradigms. In: Eggersdorfer M, Kraemer K, Cordaro JB, et al., ed. Good nutrition: perspectives for the 21st century Karger; 2016.

2. Cena H, Calder PC. Defining a healthy diet: evidence for the role of contemporary dietary patterns in health and disease. Nutrients 2020;12(2):334. DOI: 10.3390/nu12020334.

3. Schulze MB, Martínez-González MA, Fung TT, et al. Food based dietary patterns and chronic disease prevention. BMJ 2018;361:k2396. DOI: 10.1136/bmj.k2396.

4. Marlowe FW, Berbesque JC, Wood B, et al. Honey, Hadza, huntergatherers, and human evolution. J Hum Evol 2014;71:119-128. DOI: 10.1016/j.jhevol.2014.03.006.

5. Mozaffarian D, Afshin A, Benowitz NL, et al. Population approaches to improve diet, physical activity, and smoking habits. A scientific statement from the american heart association. Circulation 2012;126(12):1514-1563. DOI: 10.1161/CIR.0b013e318260a20b.

6. Tokunaga $M$, Takahashi $T$, Singh RB, et al. Diet, nutrients and noncommunicable diseases. Open Nutra J 2012;5:146-159. DOI: $10.2174 / 1876396001205010146$.

7. Moodie R, Stuckler D, Monteiro C, et al. Profits and pandemics: prevention of harmful effects of tobacco, alcohol, and ultraprocesssed food and drink industries. Lancet 2013;381(9867):670-679. DOI: 10.1016/S0140-6736(12)62089-3.

8. WHO, Burden of NCDs and their risk factors in India (Excerpted from Global Status Report on NCDs, 2014) http://www.searo.who.int/india/ topics/non-communicable_diseases/ncd_situation_global_report_ ncds_2014.pdf?ua $=1$.

9. Bantle JP, Laine DC, Castle GW, et al. Postprandial glucose and insulin responses to meals containing different carbohydrates in normal and diabetic subjects. N Engl J Med 1983;309(1):7-12. DOI: 10.1056/ NEJM198307073090102.

10. Fung TT, Brown LS. Dietary patterns and the risk of colorectal cancer. Curr Nutr Rep 2013;2(1):48-55. DOI: 10.1007/s13668-012-0031-1.

11. Estruch R, Ros E, Salas-Salvado J, et al. PREDIMED study Investigators. Primary prevention of cardiovascular disease with a mediterranean diet. N Engl J Med 2013;368(14):1279-1290. DOI: 10.1056/ NEJMoa1200303.

12. Trichopoulou A, Martinez-Gonzalez MA, Tong TY, et al. Definitions and potential health benefits of the mediterranean diet: views from experts around the world. BMC Med 2014;12:112. DOI: 10.1186/17417015-12-112. 
13. Aune D, Giovannucci E, Boffetta $P$, et al. Fruit and vegetable intake and the risk of cardiovascular disease, total cancer and all-cause mortality-a systematic review and dose-response meta-analysis of prospective studies. Int J Epidemiol 2017;46(3):1029-1056. DOI: 10.1093/ije/dyw319.

14. Du H, Li L, Bennett D, et al. China Kadoorie Biobank study. fresh fruit consumption and major cardiovascular disease in china. N Engl J Med 2016;374(14):1332-1343. DOI: 10.1056/NEJMoa1501451.

15. He FJ, Nowson CA, Lucas M, et al. Increased consumption of fruit and vegetables is related to a reduced risk of coronary heart disease: meta-analysis of cohort studies. J Hum Hypertens 2007;21(9):717-728. DOI: 10.1038/sj.jhh.1002212.

16. Poole R, Kennedy OJ, Roderick P, et al. Coffee consumption and health: umbrella review of meta-analyses of multiple health outcomes. BMJ 2017;359:j5024. DOI: 10.1136/bmj.j5024.

17. Atkinson FS, Foster-Powell K, Brand-Miller JC. International tables of glycemic index and glycemic load values: 2008. diabetes care. 2008;31(12):2281-2283.

18. Ludwig DS, Hu FB, Tappy L, et al. Dietary carbohydrates: role of quality and quantity in chronic disease. BMJ 2018;361:k2340. DOI: 10.1136/ bmj.k2340.

19. Fan J, Song $Y$, Wang $Y$, et al. Dietary glycemic index, glycemic load, and risk of coronary heart disease, stroke, and stroke mortality: a systematic review with meta-analysis. PLoS One 2012;7(12):e52182. DOI: 10.1371/journal.pone.0052182.

20. Marventano S, Vetrani $C$, Vitale $M$, et al. Whole grain intake and glycaemic control in healthy subjects: a systematic review and metaanalysis of randomized controlled trials. Nutrients 2017;9(7):E769. DOI: 10.3390/nu9070769.

21. Aune D, Keum N, Giovannucci E, et al. Whole grain consumption and risk of cardiovascular disease, cancer, and all cause and cause specific mortality: systematic review and dose-response metaanalysis of prospective studies. BMJ 2016;353:i2716. DOI: 10.1136/bmj. i2716.

22. Muraki I, Rimm EB, Willett WC, et al. Potato consumption and risk of type II diabetes: results from three prospective cohort studies. Diabetes Care 2016;39(9):376-384. DOI: 10.2337/ dc15-0547.
23. Borgi L, Rimm EB, Willett WC, et al. Potato intake and incidence of hypertension: results from three prospective US cohort studies. BMJ 2016;353:i2351. DOI: 10.1136/bmj.i2351.

24. Bazzano LA, Thompson AM, Tees MT, et al. Non-soy legume consumption lowers cholesterol levels: a meta-analysis of randomized controlled trials. Nutr Metab Cardiovasc Dis 2011;21(2):94-103. DOI: 10.1016/j.numecd.2009.08.012.

25. Marventano S, Izquierdo Pulido M, Sánchez- González C, et al. Legume consumption and CVD risk: a systematic review and meta-analysis. Public Health Nutr 2017;20(2):245-254. DOI: 10.1017/ S1368980016002299.

26. Mattei J, Hu FB, Campos H. A higher ratio of beans to white rice is associated with lower cardiometabolic risk factors in costa Rican adults. Am J Clin Nutr 2011;94(3):869-876. DOI: 10.3945/ajcn.111.013219.

27. Ramachandran A, Snehalatha C, Mary S, et al. The indian diabetes prevention programme shows that lifestyle modification and metformin prevent type II diabetes in asian indian subjects with impaired glucose tolerance (IDPP-1). Diabetologia 2006;49(2): 289-297. DOI: 10.1007/s00125-005-0097-z.

28. Schoenfeld JD, loannidis JP. Is everything we eat associated with cancer? A systematic cookbook review. Am J Clin Nutr 2013;97(1): 127-134. DOI: 10.3945/ajcn.112.047142.

29. Schulze MB, Hoffmann K. Methodological approaches to study dietary patterns in relation to risk of coronary heart disease and stroke. Br J Nutr 2006;95(5):860-869. DOI: 10.1079/BJN20061731.

30. Ley SH, Hamdy $\mathrm{O}$, Mohan V, et al. Prevention and management of type II diabetes: dietary components and nutritional strategies. Lancet 2014;383(9933):1999-2007. DOI: 10.1016/S0140-6736(14)60613-9.

31. Malik VS, Pan A, Willett WC, et al. Sugar-sweetened beverages and weight gain in children and adults: a systematic review and meta-analysis. Am J Clin Nutr 2013;98(4):1084-1102. DOI: 10.3945/ ajcn.113.058362.

32. Pereira MA, Kartashov Al, Ebbeling $C B$, et al. Fast food habits, weight gain, and insulin resistance (the CARDIA study): 15-year prospective analysis. Lancet 2005;365(9453):36-42. DOI: 10.1016/ S0140-6736(04)17663-0.

33. Azadbakht L, Esmaillzadeh A. Fast foods and risk of chronic diseases. J Res Med Sci 2008;13(1):1-2. 